\title{
Bounds for the number of points on curves over finite fields
}

\author{
Nazar Arakelian \\ CMCC, Universidade Federal do ABC, Santo André, Brazil \\ Herivelto Borges \\ ICMC, Universidade de São Paulo, São Carlos, Brazil
}

November 14, 2018

\begin{abstract}
Let $\mathcal{X}$ be a projective irreducible nonsingular algebraic curve defined over a finite field $\mathbb{F}_{q}$. This paper presents a variation of the Stöhr-Voloch theory and sets new bounds to the number of $\mathbb{F}_{q^{r-}}$ rational points on $\mathcal{X}$. In certain cases, where comparison is possible, the results are shown to improve other bounds such as Weil's, Stöhr-Voloch's and Ihara's.
\end{abstract}

\section{Introduction}

The problem of estimating the number of points on curves over finite fields has engendered a host of applications. Around 1980, Goppa presented a remarkable application of the theory of curves over finite fields, particularly those with many points, to coding theory. Elliptic-curve cryptography, created by Koblitz and Miller around 1986, is another notable application. Additional connections can be found in other areas such as finite geometry, combinatorics, and number theory.

Let $\mathcal{X}$ be a projective geometrically irreducible nonsingular algebraic curve of genus $g$ defined over a finite field $\mathbb{F}_{q}$, and let $N_{r}$ denote its number of $\mathbb{F}_{q^{r}}$-rational points. A celebrated result estimating $N_{r}$ is the Weil bound

$$
N_{r} \leq 1+q^{r}+2 g \sqrt{q^{r}}
$$

There are many examples of curves attaining Weil's bound. These are called maximal curves, and they are a significant subject in mathematical research. Nevertheless, in several cases, Weil's bound has been improved. In this regard, noteworthy results were presented by Stöhr and Voloch in 1986. Their more geometric approach provides bounds dependent on certain data of an embedding of the curve in $\mathbb{P}^{n}$. Their method not only proves Weil's bound once again, but offers substantial improvements on it in many circumstances. Accordingly, over the last few decades, researchers have used Stöhr-Voloch results as an effective tool in addressing a variety of problems related to curves over finite fields. 
A fundamental idea in the Stöhr-Voloch method is counting the number of points $P \in \mathcal{X}$ whose Frobenius image $P^{q} \in \mathcal{X}$ lies on the osculating hyperplane at $P$. The focus of this parper is a natural variation of their approach, which is counting the number points $P \in \mathcal{X}$ for which the line spanned by two different Frobenius images $P^{q^{u}}$ and $P^{q^{m}}$ intersects the $(n-2)$-th osculating space at $P$. The core of the resuts here paralell those in the original Stöhr-Voloch paper [21]. Our bounds, however, will also take into account points defined over exensions of $\mathbb{F}_{q}$, namely, $\mathbb{F}_{q^{u}}, \mathbb{F}_{q^{m}}$ and $\mathbb{F}_{q^{m-u}}$ (see Theorem 4.4). Such bounds are effective in many settings, as will be seen in Section 5. Camparison with other bounds such as Weil's, Stöhr-Voloch's and Ihara's bound is sometimes possible and, in certain cases, yields substantial improvement.

This paper is organized as follows. In Section 2, some notation and preliminary results are presented. In Section 3, for any given pair of coprime integers $u$ and $m$, with $m>u \geq 1$, and a nondegenrated $\mathbb{F}_{q^{-}}$-morphism $\mathcal{X} \stackrel{\phi}{\rightarrow} \mathbb{P}^{n}$, we associate an effective divisor $T_{u, m}$ on $\mathcal{X}$. Such divisor, which depends only on $q, u, m$ and the linear series associated to $\phi$, collects the $\mathbb{F}_{q^{r}}$-rational points on $\mathcal{X}$, for $r \in\{1, u, m, m-u\}$. Section 4 describes the core of the study. The weight of each type of point on the divisor $T_{u, m}$ is estimated and the principal result is established. Some arithmetic properties of a sequence $\left(\kappa_{0}, \ldots, \kappa_{n-2}\right)$, that will naturally arise from the data $q, u, m$ and $\phi$, are examined. Among other results, criteria for such sequence to be the classical sequence $(0, \ldots, n-2)$ is provided. Section 5 provides examples related to the new bounds and compares the results with those in the literature, such as Weil's, Stöhr-Voloch's and Ihara's bound.

\section{Notation}

The following notation will be used throughout the paper.

- $\mathbb{F}_{q}$ is the finite field with $q=p^{h}$ elements, where $p$ is a prime, and $\overline{\mathbb{F}}_{q}$ denotes its algebraic closure.

- For an irreducible curve $\mathcal{Y}$ defined over $\mathbb{F}_{q}$ and an algebraic extension $\mathbb{H}$ of $\mathbb{F}_{q}$, the function field of $\mathcal{Y}$ over $\mathbb{H}$ is denoted by $\mathbb{H}(\mathcal{Y})$.

- By a point $P \in \mathcal{Y}$, we mean a point in a nonsingular model of $\mathcal{Y}$. More precisely, the points of $\mathcal{Y}$ will be regarded as branches over $\overline{\mathbb{F}}_{q}$.

- For a curve $\mathcal{Y}$ and $r>0$, the set of its $\mathbb{F}_{q^{r}}$-rational points is denoted by $\mathcal{Y}\left(\mathbb{F}_{q^{r}}\right)$.

- $N_{r}(\mathcal{Y})$ is the number of $\mathbb{F}_{q^{r}}$-rational points of the curve $\mathcal{Y}$. If no confusion arises, $N_{r}(\mathcal{Y})$ will be simply denoted by $N_{r}$.

- For $P \in \mathcal{Y}$, the discrete valuation at $P$ is denoted by $v_{P}$.

- Given $g \in \overline{\mathbb{F}}_{q}(\mathcal{X}), t$ a separating variable of $\overline{\mathbb{F}}_{q}(\mathcal{X})$ and $r \geq 0$, the $r$-th Hasse derivative of $g$ with respect to $t$ is denoted by $D_{t}^{(r)} g$. 


\section{Preliminary results}

Let $\mathcal{X}$ be a projective geometrically irreducible nonsingular algebraic curve of genus $g$ defined over $\mathbb{F}_{q}$. Associated with the nondegenerated morphism $\phi=\left(f_{0}: \cdots: f_{n}\right): \mathcal{X} \longrightarrow \mathbb{P}^{n}\left(\overline{\mathbb{F}}_{q}\right)$, there exists a base-point-free linear series on $\mathcal{X}$ of dimension $n$ given by

$$
\mathcal{D}=\left\{\operatorname{div}\left(\sum_{i=0}^{n} a_{i} f_{i}\right)+E \mid\left(a_{0}: \ldots: a_{n}\right) \in \mathbb{P}^{n}\left(\overline{\mathbb{F}}_{q}\right)\right\},
$$

where $E:=\sum_{P \in \mathcal{X}} e_{P} P$ and $e_{P}=-\min \left\{v_{P}\left(f_{0}\right), \ldots, v_{P}\left(f_{n}\right)\right\}$. Conversely, each base-point-free linear series of dimension $n$ on $\mathcal{X}$ gives rise to a unique (up to projective transformation) nondegenerated morphism $\mathcal{X} \longrightarrow \mathbb{P}^{n}\left(\overline{\mathbb{F}}_{q}\right)$. The degree of the linear series $\mathcal{D}$ is the degree of the divisor $E$. For each point $P \in \mathcal{X}$, there exists a sequence of non-negative integers $j_{0}(P), \ldots, j_{n}(P)$, with $j_{0}(P)<\cdots<j_{n}(P)$, called ordersequence at $P$ with respect to $\mathcal{D}$. The integers $j \geq 0$ defining this sequence are those for which $v_{P}(D)=j$ for some $D \in \mathcal{D}$, and they are called $(\mathcal{D}, P)$-orders. Since $\mathcal{D}$ is base-point-free, $j_{0}(P)=0$ for all $P \in \mathcal{X}$. When there is no risk of confusion, $j_{i}$ denotes $j_{i}(P)$. Let $L_{i}(P)$ be the intersection of all hyperplanes in $\mathbb{P}^{n}\left(\overline{\mathbb{F}}_{q}\right)$ that intersect $\mathcal{X}$ at $P$ with multiplicity at least $j_{i+1}$. The space $L_{i}(P)$ is called the $i$-th osculating space at $P$. The $(n-2)$-th osculating spaces will be of particular interest in the next section, and so will be the following, as proved in [21, Theorem 1.1].

Theorem 2.1. Let $P \in \mathcal{X}$ and $t$ be a local parameter at $P$. Suppose that $e_{P}=0$. Assume that the first $i(\mathcal{D}, P)$-orders $j_{0}, \ldots, j_{i-1}$ are known. Then $j_{i}$ is the smallest integer such that the points $\left(\left(D_{t}^{\left(j_{s}\right)} f_{0}\right)(P): \cdots:\left(D_{t}^{\left(j_{s}\right)} f_{n}\right)(P)\right)$ with $s=0, \ldots, i$ are linearly independent, and $L_{i}(P)$ is spanned by these points.

All but finitely many points $P \in \mathcal{X}$ have the same order-sequence (see e.g. [21, Theorem 1.5]), denoted by $\left(\epsilon_{0}, \ldots, \epsilon_{n}\right)$, which is called the order-sequence of $\mathcal{X}$ with respect to $\mathcal{D}$. This sequence can also be defined as the minimal sequence, with respect to the lexicographic order, for which

$$
\operatorname{det}\left(D_{t}^{\left(\epsilon_{i}\right)} f_{j}\right)_{0 \leq i, j \leq n} \neq 0
$$

where $t \in \overline{\mathbb{F}}_{q}(\mathcal{X})$ is a separating variable. Moreover, for each $P \in \mathcal{X}$,

$$
\epsilon_{i} \leq j_{i}(P) \text { for all } i \in\{0, \ldots, n\}
$$

The curve $\mathcal{X}$ is called classical with respect to $\phi$ (or $\mathcal{D})$ if $\left(\epsilon_{0}, \ldots, \epsilon_{n}\right)=(0, \ldots, n)$ and is called nonclassical otherwise.

Now assume that $\phi$ is defined over $\mathbb{F}_{q}$. The sequence of non-negative integers $\left(\nu_{0}, \ldots, \nu_{n-1}\right)$, chosen 
minimally in the lexicographic order, such that

$$
\left|\begin{array}{ccc}
f_{0}^{q} & \cdots & f_{n}^{q} \\
D_{t}^{\left(\nu_{0}\right)} f_{0} & \cdots & D_{t}^{\left(\nu_{0}\right)} f_{n} \\
\vdots & \cdots & \vdots \\
D_{t}^{\left(\nu_{n-1}\right)} f_{0} & \cdots & D_{t}^{\left(\nu_{n-1}\right)} f_{n}
\end{array}\right| \neq 0
$$

where $t \in \mathbb{F}_{q}(\mathcal{X})$ is a separating variable, is called the $\mathbb{F}_{q}$-Frobenius order-sequence of $\mathcal{X}$ with respect to $\phi$. From [21, Proposition 2.1], we have that $\left\{\nu_{0}, \ldots, \nu_{n-1}\right\}=\left\{\epsilon_{0}, \ldots, \epsilon_{n}\right\} \backslash\left\{\epsilon_{I}\right\}$ for some $I \in\{1, \ldots, n\}$. If $\left(\nu_{0}, \ldots, \nu_{n-1}\right)=(0, \ldots, n-1)$, then $\mathcal{X}$ is called $\mathbb{F}_{q}$-Frobenius classical with respect to $\phi$. Otherwise, $\mathcal{X}$ is called $\mathbb{F}_{q}$-Frobenius nonclassical. The following result follows from [3, Theorem 1.1].

Theorem 2.2. Let $m$ and $u$ be coprime integers, with $m>u \geq 1$, and consider the plane curve $\mathcal{F}_{m, u}: f(x, y)=0$, where $f(x, y) \in \mathbb{F}_{q}[x, y]$ is the polynomial

$$
f(x, y)=\frac{\left(x^{q^{u}}-x\right)\left(y^{q^{m}}-y\right)-\left(x^{q^{m}}-x\right)\left(y^{q^{u}}-y\right)}{\left(x^{q^{2}}-x\right)\left(y^{q}-y\right)-\left(x^{q}-x\right)\left(y^{q^{2}}-y\right)} .
$$

Then $\mathcal{F}_{m, u}$ is the only simultaneously $\mathbb{F}_{q^{m}}$ - and $\mathbb{F}_{q^{u}}$-Frobenius nonclassical curve for the morphism of lines. It has $\mathcal{F}_{m, u}\left(\mathbb{F}_{q}\right)=\emptyset$ and order-sequence $\left(0,1, q^{u}\right)$. In particular, $x \in \mathbb{F}_{q}\left(\mathcal{F}_{m, u}\right)$ is a separating variable and

$$
\left|\begin{array}{ccc}
1 & x^{q^{u}} & y^{q^{u}} \\
1 & x & y \\
D_{x}^{\left(q^{u}\right)} 1 & D_{x}^{\left(q^{u}\right)} x & D_{x}^{\left(q^{u}\right)} y
\end{array}\right| \cdot\left|\begin{array}{ccc}
1 & x^{q^{m}} & y^{q^{m}} \\
1 & x & y \\
D_{x}^{\left(q^{u}\right)} 1 & D_{x}^{\left(q^{u}\right)} x & D_{x}^{\left(q^{u}\right)} y
\end{array}\right| \neq 0
$$

and

$$
\left|\begin{array}{ccc}
1 & x^{q^{u}} & y^{q^{u}} \\
1 & x & y \\
D_{x}^{(i)} 1 & D_{x}^{(i)} x & D_{x}^{(i)} y
\end{array}\right|=\left|\begin{array}{ccc}
1 & x^{q^{m}} & y^{q^{m}} \\
1 & x & y \\
D_{x}^{(i)} 1 & D_{x}^{(i)} x & D_{x}^{(i)} y
\end{array}\right|=0 \text { for } i \in\left\{0,1, \ldots, q^{u}-1\right\}
$$

Based on the proof of [21, Theorem 1.5], one can conclude the following:

Proposition 2.3. Consider $P \in \mathcal{X}$ and let $t$ be a local parameter at $P$. For each $i \in\{0, \ldots, e\}$, let $z_{i} \in \overline{\mathbb{F}}_{q}(\mathcal{X})$ be such that $z_{i}=t^{j_{i}}+\sum_{k=j_{i}+1}^{\infty} a_{k} t^{k}$ with $j_{0}<j_{1}<\cdots<j_{e}$, where $e>0$ is an integer. Then, for $0 \leq m_{0}<\cdots<m_{e}$,

$$
v_{P}\left(\operatorname{det}\left(D_{t}^{\left(m_{s}\right)} z_{r}\right)_{0 \leq r, s \leq e}\right) \geq \sum_{i=0}^{e}\left(j_{i}-m_{i}\right)
$$

and the equality holds if and only if $p \nmid \operatorname{det}\left(\left(\begin{array}{c}j_{r} \\ m_{s}\end{array}\right)\right)_{0 \leq r, s \leq e}$ 


\section{The $\left(q^{u}, q^{m}\right)$-Frobenius divisor}

Hereafter, $\mathcal{X}$ will denote a projective geometrically irreducible nonsingular algebraic curve of genus $g$ defined over $\mathbb{F}_{q}$. In addition, unless otherwise stated, the morphisms

$$
\phi=\left(f_{0}: \ldots: f_{n}\right): \mathcal{X} \longrightarrow \mathbb{P}^{n}\left(\overline{\mathbb{F}}_{q}\right)
$$

will always be nondegenerated, defined over $\mathbb{F}_{q}$, and $n \geq 2$. Let $m$ and $u$ be coprime integers, with $m>u \geq 1$, and consider the Frobenius maps $\Phi_{q^{i}}: \mathcal{X} \longrightarrow \mathcal{X}, i \in\{u, m\}$. We want to estimate the number of points $P \in \mathcal{X}$ for which a line through $\Phi_{q^{u}}(P)$ and $\Phi_{q^{m}}(P)$ intersects the osculating space $L_{n-2}(P)$. This and Theorem 2.1 lead us to study the functions

$$
\mathcal{A}_{t}^{\rho_{0}, \ldots, \rho_{n-2}}\left(f_{0}, \ldots, f_{n}\right):=\left|\begin{array}{cccc}
f_{0}^{q^{m}} & f_{1}^{q^{m}} & \cdots & f_{n}^{q^{m}} \\
f_{0}^{q^{u}} & f_{1}^{q^{u}} & \cdots & f_{n}^{q^{u}} \\
D_{t}^{\left(\rho_{0}\right)} f_{0} & D_{t}^{\left(\rho_{0}\right)} f_{1} & \cdots & D_{t}^{\left(\rho_{0}\right)} f_{n} \\
\vdots & \vdots & \cdots & \vdots \\
D_{t}^{\left(\rho_{n-2}\right)} f_{0} & D_{t}^{\left(\rho_{n-2}\right)} f_{1} & \cdots & D_{t}^{\left(\rho_{n-2}\right)} f_{n}
\end{array}\right|,
$$

where $t \in \mathbb{F}_{q}(\mathcal{X})$ is a separating variable, and $\rho_{0}, \ldots, \rho_{n-2}$ are non-negative integers.

In what follows, $\left(\nu_{0}, \ldots, \nu_{n-1}\right)$ and $\left(\mu_{0}, \ldots, \mu_{n-1}\right)$ will be the $\mathbb{F}_{q^{u}}$ and the $\mathbb{F}_{q^{m}}$-Frobenius ordersequences, respectively, on $\mathcal{X}$ associated to $\mathcal{D}$.

Proposition 3.1. There exist integers $\kappa_{0}, \ldots, \kappa_{n-2}$, with $0 \leq \kappa_{0}<\cdots<\kappa_{n-2}$, such that $\mathcal{A}_{t}^{\kappa_{0}, \ldots, \kappa_{n-2}}\left(f_{0}, \ldots, f_{n}\right) \neq 0$. Choose them minimally in the lexicographic order. Then there exist $I, J \in$ $\{0, \ldots, n-1\}$ such that

$$
\left\{\nu_{0}, \ldots, \nu_{n-1}\right\} \backslash\left\{\nu_{I}\right\}=\left\{\kappa_{0}, \ldots, \kappa_{n-2}\right\}=\left\{\mu_{0}, \ldots, \mu_{n-1}\right\} \backslash\left\{\mu_{J}\right\} .
$$

Proof. For $i \in\{m, n\}$ and $j \in\left\{\nu_{0}, \ldots, \nu_{n-1}\right\}$, set $\Phi_{q^{i}}(\phi):=\left(f_{0}^{q^{i}}, \ldots, f_{n}^{q^{i}}\right)$ and $D_{t}^{(j)}(\phi):=\left(D_{t}^{(j)} f_{0}, \ldots, D_{t}^{(j)} f_{n}\right)$ to represent the vectors. It is clear that $\Phi_{q^{u}}(\phi)$ and $\Phi_{q^{m}}(\phi)$ are linearly independent, and (2.2) gives the linear independence of vectors $\Phi_{q^{u}}(\phi), D_{t}^{\left(\nu_{0}\right)}(\phi), \ldots, D_{t}^{\left(\nu_{n-1}\right)}(\phi)$. Now if the $n+1$ vectors

$$
\Phi_{q^{m}}(\phi), \Phi_{q^{u}}(\phi), D_{t}^{\left(\nu_{0}\right)}(\phi), \ldots, D_{t}^{\left(\nu_{n-2}\right)}(\phi)
$$

are linearly independent over $\mathbb{F}_{q}(\mathcal{X})$, then the result follows. Otherwise, let $I \in\{0, \ldots, n-2\}$ be the smallest integer for which $\Phi_{q^{m}}(\phi)$ is a linear combination of $\Phi_{q^{u}}(\phi), D_{t}^{\left(\nu_{0}\right)}(\phi), \ldots, D_{t}^{\left(\nu_{I}\right)}(\phi)$, and note that the linear independence of $\Phi_{q^{u}}(\phi), D_{t}^{\left(\nu_{0}\right)}(\phi), \ldots, D_{t}^{\left(\nu_{n-1}\right)}(\phi)$ implies that of

$$
\left\{\Phi_{q^{m}}(\phi), \Phi_{q^{u}}(\phi), D_{t}^{\left(\nu_{0}\right)}(\phi), \ldots, D_{t}^{\left(\nu_{n-1}\right)}(\phi)\right\} \backslash\left\{D_{t}^{\left(\nu_{I}\right)}(\phi)\right\}
$$


Therefore, we clearly have $\left\{\kappa_{0}, \ldots, \kappa_{n-2}\right\}=\left\{\nu_{0}, \ldots, \nu_{n-1}\right\} \backslash\left\{\nu_{I}\right\}$, and the same argument implies $\left\{\kappa_{0}, \ldots, \kappa_{n-2}\right\}=$ $\left\{\mu_{0}, \ldots, \mu_{n-1}\right\} \backslash\left\{\mu_{J}\right\}$.

Hereafter, the sequence $\left(\kappa_{0}, \ldots, \kappa_{n-2}\right)$, given in Proposition 3.1, will be called the $\left(q^{u}, q^{m}\right)$-Frobenius order-sequence of $\mathcal{X}$ with respect to $\mathcal{D}$ (or $\phi)$. The curve $\mathcal{X}$ will be called $\left(q^{u}, q^{m}\right)$-Frobenius classical with respect to $\mathcal{D}$ when $\kappa_{i}=i$ for all $i \in\{0,1, \ldots, n-2\}$. Otherwise, $\mathcal{X}$ will be called $\left(q^{u}, q^{m}\right)$-Frobenius nonclassical. Note that $\kappa_{n-2} \leq \epsilon_{n} \leq d=\operatorname{deg}(\mathcal{D})$.

We shall omit the proof of the following result, as it is analogous to [21, Proposition 2.2].

Proposition 3.2. (a) If $g_{i}=\sum a_{i j} f_{j}$ with $\left(a_{i j}\right) \in G L_{n+1}\left(\mathbb{F}_{q}\right)$, then

$$
\mathcal{A}_{t}^{\kappa_{0}, \ldots, \kappa_{n-2}}\left(g_{0}, \ldots, g_{n}\right)=\operatorname{det}\left(a_{i j}\right) \cdot \mathcal{A}_{t}^{\kappa_{0}, \ldots, \kappa_{n-2}}\left(f_{0}, \ldots, f_{n}\right)
$$

(b) If $h \in \mathbb{F}_{q}(\mathcal{X})^{*}$, then

$$
\mathcal{A}_{t}^{\kappa_{0}, \ldots, \kappa_{n-2}}\left(h f_{0}, \ldots, h f_{n}\right)=h^{q^{m}+q^{u}+n-1} \cdot \mathcal{A}_{t}^{\kappa_{0}, \ldots, \kappa_{n-2}}\left(f_{0}, \ldots, f_{n}\right) .
$$

(c) If $x \in \mathbb{F}_{q}(\mathcal{X})$ is another separating variable, then

$$
\mathcal{A}_{x}^{\kappa_{0}, \ldots, \kappa_{n-2}}\left(f_{0}, \ldots, f_{n}\right)=\left(\frac{d t}{d x}\right)^{\kappa_{0}+\kappa_{1}+\cdots+\kappa_{n-2}} \cdot \mathcal{A}_{t}^{\kappa_{0}, \ldots, \kappa_{n-2}}\left(f_{0}, \ldots, f_{n}\right) .
$$

Definition 3.3. The $\left(q^{u}, q^{m}\right)$-Frobenius divisor of $\mathcal{D}$ is defined by

$$
T_{u, m}=\operatorname{div}\left(\mathcal{A}_{t}^{\kappa_{0}, \ldots, \kappa_{n-2}}\left(f_{0}, \ldots, f_{n}\right)\right)+\left(\kappa_{0}+\kappa_{1}+\cdots+\kappa_{n-2}\right) \operatorname{div}(d t)+\left(q^{m}+q^{u}+n-1\right) E,
$$

where $t \in \mathbb{F}_{q}(\mathcal{X})$ is a separating variable and $E=\sum_{P \in \mathcal{X}} e_{P} P$ for $e_{P}=-\min \left\{v_{P}\left(f_{0}\right), \ldots, v_{P}\left(f_{n}\right)\right\}$.

Note that given $u$ and $m$, Proposition 3.2 implies that the sequence $\left(\kappa_{0}, \ldots, \kappa_{n-2}\right)$, as well as the divisor $T_{u, m}$, depend only on the linear series $\mathcal{D}$. In addition,

$$
\operatorname{deg}\left(T_{u, m}\right)=\left(\kappa_{0}+\kappa_{1}+\cdots+\kappa_{n-2}\right)(2 g-2)+\left(q^{m}+q^{u}+n-1\right) d,
$$

where $d:=\operatorname{deg}(\mathcal{D})=\operatorname{deg}(E)$.

Based on Propostion 3.2 and Definition 3.3 for any given $P \in \mathcal{X}$, we can assume $e_{P}=0$ before computing $v_{P}\left(T_{u, m}\right)$. This will be done systematically, unless otherwise noted.

Proposition 3.4. Let $\phi=\left(f_{0}: \cdots: f_{n}\right): \mathcal{X} \longrightarrow \mathbb{P}^{n}\left(\overline{\mathbb{F}}_{q}\right)$ be a morphism defined over $\mathbb{F}_{q}$ and let $\left(\kappa_{0}, \ldots, \kappa_{n-2}\right)$ be its $\left(q^{u}, q^{m}\right)$-Frobenius order-sequence. If $\kappa_{0}>0$, then $m>n \geq 2$ and the following hold. 
(i) The field $\mathbb{F}_{q}(\phi(\mathcal{X}))$ is $\mathbb{F}_{q}$-isomorphic to an intermediate field of $N / \mathbb{F}_{q}\left(\mathcal{F}_{u, m}\right)$, where $\mathcal{F}_{u, m}$ is the curve in Theorem 2.2 and $N$ is the Galois closure of $\mathbb{F}_{q}\left(\mathcal{F}_{u, m}\right) / \mathbb{F}_{q}(x)$ in a fixed algebraic closure of $\mathbb{F}_{q}(x)$.

(ii) There exist only a finite number of curves $\mathcal{X}$ (up to $\mathbb{F}_{q}$-isomorphism) that admit a projective model for which $\kappa_{0}>0$.

(iii) If $n=2$, then $\phi(\mathcal{X})$ is isomorphic to $\mathcal{F}_{u, m}$.

(iv) $\kappa_{0}=q^{u}$.

(v) $\mathcal{X}\left(\mathbb{F}_{q}\right)=\emptyset$.

Proof. Assume $f_{0}=1$ and set $x:=f_{1}$. In particular, $\mathbb{F}_{q}(\phi(\mathcal{X}))=\mathbb{F}_{q}\left(x, f_{2}, \ldots, f_{n}\right)$. Since $\kappa_{0}>0$, the matrix

$$
\left(\begin{array}{ccccc}
1 & x^{q^{m}} & f_{2}^{q^{m}} & \cdots & f_{n}^{q^{m}} \\
1 & x^{q^{u}} & f_{2}^{q^{u}} & \cdots & f_{n}^{q^{u}} \\
1 & x & f_{2} & \cdots & f_{n}
\end{array}\right)
$$

has rank 2 , and then, $f_{2}, \ldots, f_{n}$ are roots of $\left(x^{q^{m}}-x\right)\left(T^{q^{u}}-T\right)-\left(T^{q^{m}}-T\right)\left(x^{q^{u}}-x\right) \in \mathbb{F}_{q}(x)[T]$. As $\phi$ is nondegenerated, the set $\left\{a_{0}+a_{1} x+a_{2} f_{2}+\cdots+a_{n} f_{n} \mid a_{i} \in \mathbb{F}_{q}\right\}$ has $q^{n+1}$ distinct roots of the above polynomial, which gives $m>n \geq 2$. In addtion, since $f_{2}, \ldots, f_{n}$ are roots of the separable polynomial

$$
h(x, T)=\frac{\left(x^{q^{m}}-x\right)\left(T^{q^{u}}-T\right)-\left(T^{q^{m}}-T\right)\left(x^{q^{u}}-x\right)}{\left(x^{q^{2}}-x\right)\left(T^{q}-T\right)-\left(T^{q^{2}}-T\right)\left(x^{q}-x\right)} \in \mathbb{F}_{q}(x)[T],
$$

assertions (i), (ii), and (iii) immediately follow from Theorem 2.2. To prove (iv), note that $x \in \mathbb{F}_{q}(\phi(\mathcal{X}))$ is a separating variable, and then the matrix

$$
\left(\begin{array}{ccccc}
1 & x^{q^{m}} & f_{2}^{q^{m}} & \cdots & f_{n}^{q^{m}} \\
1 & x^{q^{u}} & f_{2}^{q^{u}} & \cdots & f_{n}^{q^{u}} \\
0 & D_{x}^{\left(\kappa_{0}\right)} x & D_{x}^{\left(\kappa_{0}\right)} f_{2} & \cdots & D_{x}^{\left(\kappa_{0}\right)} f_{n}
\end{array}\right)
$$

has rank 3. Thus for some $y \in\left\{f_{2}, \ldots, f_{n}\right\}$, we have

$$
\left|\begin{array}{ccc}
1 & x^{q^{m}} & y^{q^{m}} \\
1 & x^{q^{u}} & y^{q^{u}} \\
0 & D_{x}^{\left(\kappa_{0}\right)} x & D_{x}^{\left(\kappa_{0}\right)} y
\end{array}\right| \neq 0
$$

and the minimality of $\kappa_{0}>0$ gives

$$
\left|\begin{array}{ccc}
1 & x^{q^{m}} & y^{q^{m}} \\
1 & x^{q^{u}} & y^{q^{u}} \\
D_{x}^{(i)} 1 & D_{x}^{(i)} x & D_{x}^{(i)} y
\end{array}\right|=0 \text { for } i \in\left\{0, \ldots, \kappa_{0}-1\right\}
$$


In addition, $\kappa_{0}>0$ implies

$$
0=\left|\begin{array}{ccc}
1 & x^{q^{m}} & y^{q^{m}} \\
1 & x^{q^{u}} & y^{q^{u}} \\
D_{x}^{(i)} 1 & D_{x}^{(i)} x & D_{x}^{(i)} y
\end{array}\right| \Longleftrightarrow\left|\begin{array}{ccc}
1 & x^{q^{m}} & y^{q^{m}} \\
1 & x & y \\
D_{x}^{(i)} 1 & D_{x}^{(i)} x & D_{x}^{(i)} y
\end{array}\right|=\left|\begin{array}{ccc}
1 & x^{q^{u}} & y^{q^{u}} \\
1 & x & y \\
D_{x}^{(i)} 1 & D_{x}^{(i)} x & D_{x}^{(i)} y
\end{array}\right|=0
$$

for all $i \geq 0$. Therefore, (2.3) and (2.4) in Theorem 2.2 together with (3.5) and (3.7), imply $\kappa_{0}=q^{u}$. Finally, note that the case $i=0$ in (3.6) implies $h(x, y)=0$. That is, there exists a copy of the curve $\mathcal{F}_{u, m}$ being $\mathbb{F}_{q}$-covered by $\mathcal{X}$. Since $\mathcal{F}_{u, m}\left(\mathbb{F}_{q}\right)=\emptyset$ (Theorem 2.2), we obtain $(v)$.

Remark 3.5. In view of Proposition 3.4, from now on, unless otherwise stated, we always assume that $\kappa_{0}=0$.

Let us establish some important properties of the $\left(q^{u}, q^{m}\right)$-Frobenius order-sequence, beginning with the following result, whose proof is straightforward.

Proposition 3.6. If $m_{0}, \ldots, m_{s}$ are integers with $0 \leq m_{0}<\cdots<m_{s}$ such that the rows of the matrix

$$
\left(\begin{array}{cccc}
f_{0}^{q^{m}} & f_{1}^{q^{m}} & \cdots & f_{n}^{q^{m}} \\
f_{0}^{q^{u}} & f_{1}^{q^{u}} & \cdots & f_{n}^{q^{u}} \\
D_{t}^{\left(m_{0}\right)} f_{0} & D_{t}^{\left(m_{0}\right)} f_{1} & \cdots & D_{t}^{\left(m_{0}\right)} f_{n} \\
\vdots & \vdots & \ddots & \vdots \\
D_{t}^{\left(m_{s}\right)} f_{0} & D_{t}^{\left(m_{s}\right)} f_{1} & \cdots & D_{t}^{\left(m_{s}\right)} f_{n}
\end{array}\right)
$$

are linearly independent over $\mathbb{F}_{q}(\mathcal{X})$, then $\kappa_{i} \leq m_{i}$ for each $i \in\{0, \ldots, s\}$.

Theorem 3.7. Let $\phi=\left(f_{0}: f_{1}: \cdots: f_{n}\right): \mathcal{X} \longrightarrow \mathbb{P}^{n}\left(\overline{\mathbb{F}}_{q}\right)$ with $n>2$ be a morphism defined over $\mathbb{F}_{q}$ such that $\mathcal{X}$ is $\left(q^{u}, q^{m}\right)$-Frobenius nonclassical with respect to $\phi$. Let $\ell \in\{1, \ldots, n-2\}$ be such that $\kappa_{i}=i$ for $i<\ell$ and $\kappa_{\ell}>\ell$. Then $p \mid \kappa_{\ell}$.

Proof. Let $t \in \mathbb{F}_{q}(\mathcal{X})$ be a separating variable. Consider the sequence $\left(m_{0}, \ldots, m_{n-2}\right)$ with $m_{i}=\kappa_{i}$ for $i \in$ $\{0,1, \ldots, n-2\} \backslash\{\ell\}$ and $m_{\ell}=\kappa_{\ell}-1$. Since $m_{\ell}<\kappa_{\ell}$, Proposition $3.6 \operatorname{implies} \mathcal{A}_{t}^{m_{0}, \ldots, m_{n-2}}\left(f_{0}, \ldots, f_{n}\right)=0$.

Thus it suffices to show that

$$
D_{t}^{(1)}\left(\mathcal{A}_{t}^{m_{0}, \ldots, m_{n-2}}\left(f_{0}, \ldots, f_{n}\right)\right)=\kappa_{\ell} \cdot \mathcal{A}_{t}^{\left(\kappa_{0}, \ldots, \kappa_{n-2}\right)}\left(f_{0}, \ldots, f_{n}\right) .
$$

Let $S_{n+1}$ denote the group of permutations on $\{0,1, \ldots, n\}$. We have that

$$
\mathcal{A}_{t}^{m_{0}, \ldots, m_{n-2}}\left(f_{0}, \ldots, f_{n}\right)=\sum_{\sigma \in S_{n+1}}\left(\operatorname{sign}(\sigma) \cdot f_{\sigma(0)}^{q^{m}} \cdot f_{\sigma(1)}^{q^{u}} \cdot\left(D_{t}^{\left(m_{0}\right)} f_{\sigma(2)}\right) \cdots\left(D_{t}^{\left(m_{n-2}\right)} f_{\sigma(n)}\right)\right) .
$$


Therefore, $D_{t}^{(1)}\left(\mathcal{A}_{t}^{m_{0}, \ldots, m_{n-2}}\left(f_{0}, \ldots, f_{n}\right)\right)$ is the sum over all $\sigma \in S_{n+1}$ of

$$
\operatorname{sign}(\sigma) \cdot f_{\sigma(0)}^{q^{m}} \cdot f_{\sigma(1)}^{q^{u}} \cdot D_{t}^{(1)}\left(\left(D_{t}^{\left(m_{0}\right)} f_{\sigma(2)}\right) \cdots\left(D_{t}^{\left(m_{n-2}\right)} f_{\sigma(n)}\right)\right)
$$

In addition, for any fixed $i \in\{0, \ldots, n-2\}$, the sum over all $\sigma \in S_{n+1}$ of the terms

$$
\operatorname{sign}(\sigma) \cdot f_{\sigma(0)}^{q^{m}} \cdot f_{\sigma(1)}^{q^{u}} \cdot\left(D_{t}^{\left(m_{0}\right)} f_{\sigma(2)}\right) \cdots \underbrace{\left(D_{t}^{(1)}\left(D_{t}^{\left(m_{i}\right)} f_{\sigma(i+2)}\right)\right)}_{\left(m_{i}+1\right) \cdot D_{t}^{\left(m_{i}+1\right)} f_{\sigma(i+2)}} \cdots\left(D_{t}^{\left(m_{n-2}\right)} f_{\sigma(n)}\right)
$$

will give rise to the determinant corresponding to $\left(m_{i}+1\right) \cdot \mathcal{A}_{t}^{m_{0}, \ldots, m_{i}+1, \ldots, m_{n-2}}\left(f_{0}, \ldots, f_{n}\right)$. From Proposition [3.6], the latter determinants vanish if $i \neq \ell$, and we arrive at (3.9).

The following corollaries are immediate consequences of Theorem 3.7

Corollary 3.8. If $p>\operatorname{deg}(\mathcal{D})$, then $\mathcal{X}$ is $\left(q^{u}, q^{m}\right)$-Frobenius classical.

Corollary 3.9. Assume $p>n-1$. If $\mathcal{X}$ is $\left(q^{u}, q^{m}\right)$-Frobenius nonclassical w.r.t. $\phi$, then $\mathcal{X}$ is $\mathbb{F}_{q^{r-}}$ Frobenius nonclassical for $r \in\{u, m\}$. Moreover, if $p>n$, then $\mathcal{X}$ is nonclassical.

\section{Upper bounds for the number of rational points}

This section provides the main results of this paper. Consider a point $P \in \mathcal{X}$, and let $t$ be a local parameter at $P$. Since $v_{P}(\operatorname{div}(d t))=0$ and we assume $e_{P}=0$, it follows that

$$
v_{P}\left(T_{u, m}\right)=v_{P}\left(\mathcal{A}_{t}^{\kappa_{0}, \ldots, \kappa_{n-2}}\left(f_{0}, \ldots, f_{n}\right)\right) \geq 0 .
$$

That is, $T_{u, m}$ is an effective divisor. Moreover, because any $P \in \mathcal{X}\left(\mathbb{F}_{q^{r}}\right)$ with $r \in\{u, m, m-u\}$ will render the three first rows of $\mathcal{A}_{t}^{\kappa_{0}, \ldots, \kappa_{n-2}}\left(f_{0}, \ldots, f_{n}\right)$ linearly dependent (cf. Remark 3.5), it follows that $v_{P}\left(T_{u, m}\right) \geq 1$ for all such points. The next result refines these bounds.

Proposition 4.1. Let $P \in \mathcal{X}$ with $(\mathcal{D}, P)$-orders $j_{0}, \ldots, j_{n}$. Then

$$
v_{P}\left(T_{u, m}\right) \geq\left\{\begin{array}{l}
j_{1} q^{u}+\sum_{i=0}^{n-2}\left(j_{i+2}-\kappa_{i}\right) \text { if } P \in \mathcal{X}\left(\mathbb{F}_{q}\right) ; \\
j_{1} q^{u}+\sum_{i=0}^{n-2}\left(j_{i}-\kappa_{i}\right) \text { for } P \in \mathcal{X}\left(\mathbb{F}_{q^{m-u}}\right) ; \\
\sum_{i=1}^{n-1}\left(j_{i}-\kappa_{i-1}\right), \text { if } P \in \mathcal{X}\left(\mathbb{F}_{q^{r}}\right), \text { for } r \in\{u, m\} \\
\sum_{i=0}^{n-2}\left(j_{i}-\kappa_{i}\right) \text { for arbitrary point } P \in \mathcal{X} .
\end{array}\right.
$$

In addition, 
(i) Equality holds in (4.1) if and only if $p \nmid \operatorname{det}\left(\left(\begin{array}{c}j_{i} \\ \kappa_{s}\end{array}\right)\right)_{2 \leq i \leq n, 0 \leq s \leq n-2}$.

(ii) If $p \mid \operatorname{det}\left(\left(\begin{array}{c}j_{i} \\ \kappa_{s}\end{array}\right)\right)_{1 \leq i \leq n-1,0 \leq s \leq n-2}$, then the strict inequality holds in both cases of (4.3).

(iii) If $p \mid \operatorname{det}\left(\left(\begin{array}{c}j_{i} \\ \kappa_{s}\end{array}\right)\right)_{0 \leq i, s \leq n-2}$, then the strict inequality holds in (4.2) and (4.4)

Proof. Consider a point $P \in \mathcal{X}$, and let $t$ be a local parameter at $P$. After a suitable projective transformation $\left(a_{i j}\right) \in G L_{n+1}\left(\overline{\mathbb{F}}_{q}\right)$, we have that $g_{i}:=\sum_{j=0}^{n} a_{i j} f_{i}$ has a local expansion $g_{i}=t^{j_{i}}+\cdots$, for $i=0, \ldots, n$, where the dots indicate terms of higher order. Then

$$
\mathcal{A}_{t}^{\kappa_{0}, \ldots, \kappa_{n-2}}\left(f_{0}, \ldots, f_{n}\right) \cdot \operatorname{det}\left(a_{i j}\right)=\left|\begin{array}{cccc}
b_{0} & b_{1} & \ldots & b_{n} \\
h_{0} & h_{1} & \ldots & h_{n} \\
D_{t}^{\left(\kappa_{0}\right)} g_{0} & D_{t}^{\left(\kappa_{0}\right)} g_{1} & \ldots & D_{t}^{\left(\kappa_{0}\right)} g_{n} \\
D_{t}^{\left(\kappa_{1}\right)} g_{0} & D_{t}^{\left(\kappa_{1}\right)} g_{1} & \ldots & D_{t}^{\left(\kappa_{1}\right)} g_{n} \\
\vdots & \vdots & \vdots & \vdots \\
D_{t}^{\left(\kappa_{n-2}\right)} g_{0} & D_{t}^{\left(\kappa_{n-2}\right)} g_{1} & \ldots & D_{t}^{\left(\kappa_{n-2}\right)} g_{n}
\end{array}\right|,
$$

where $b_{i}=\sum_{j=0}^{n} a_{i j} f_{j}^{q^{m}}$ and $h_{i}=\sum_{j=0}^{n} a_{i j} f_{j}^{q^{u}}$. Thus

$$
\mathcal{A}_{t}^{\kappa_{0}, \ldots, \kappa_{n-2}}\left(f_{0}, \ldots, f_{n}\right) \cdot \operatorname{det}\left(a_{i j}\right)=\sum_{0 \leq l<k \leq n}(-1)^{l+k+1} \gamma_{l k} \beta_{k l},
$$

where $\beta_{k l}$ denotes the $(n-1) \times(n-1)$ minor obtained by omitting the first two rows and the $k$-th and $l$-th columns of the matrix above and $\gamma_{l k}=b_{l} h_{k}-h_{l} b_{k}$. From (2.5),

$$
v_{P}\left(\beta_{k l}\right) \geq \sum_{\delta=0}^{n-2}\left(j_{\delta}-\kappa_{\delta}\right)+j_{n-1}+j_{n}-j_{l}-j_{k}
$$

and equality holds if and only if

$$
p \nmid \operatorname{det}\left(\left(\begin{array}{c}
j_{r} \\
\kappa_{s}
\end{array}\right)\right)_{r \notin\{l, k\}} .
$$

In particular,

$$
v_{P}\left(T_{u, m}\right) \geq \min _{0 \leq l<k \leq n}\left\{v_{P}\left(\gamma_{l k}\right)+\sum_{\delta=0}^{n-2}\left(j_{\delta}-\kappa_{\delta}\right)+j_{n-1}+j_{n}-j_{l}-j_{k}\right\}
$$

We only keep track of the pairs $(l, k)$ that will minimize the right hand side of (4.8). Observe that if $P \in \mathcal{X}\left(\mathbb{F}_{q^{u}}\right)$, then we can take $\left(a_{i j}\right) \in G L_{n+1}\left(\overline{\mathbb{F}}_{q}\right)$ defined over $\mathbb{F}_{q^{u}}$, and then $h_{i}=g_{i}^{q^{u}}$ for $i \in\{0, \ldots, n\}$. The case $P \in \mathcal{X}\left(\mathbb{F}_{q^{m}}\right)$ is clearly similar. In particular, for $P \in \mathcal{X}\left(\mathbb{F}_{q}\right)$, we have $h_{i}=g_{i}^{q^{u}}$ and $b_{i}=g_{i}^{q^{m}}$ for $i \in\{0, \ldots, n\}$. This observation gives us

1) $v_{P}\left(\gamma_{l k}\right) \geq 0$ for arbitrary $P \in \mathcal{X}$, and then $(l, k)=(n-1, n)$. 
2) $v_{P}\left(\gamma_{l k}\right) \geq j_{l} q^{u}$ for $P \in \mathcal{X}$ defined over $\mathbb{F}_{q^{u}}$ or $\mathbb{F}_{q^{m}}$, and then $(l, k)=(0, n)$.

3) $v_{P}\left(\gamma_{l k}\right) \geq j_{l} q^{m}+j_{k} q^{u}$ for $P \in \mathcal{X}\left(\mathbb{F}_{q}\right)$, and then $(l, k)=(0,1)$.

Therefore, (4.8) together with 1), 2) and 3) give the lower bounds in (4.4), (4.3) and (4.1), respectively.

For $P \in \mathcal{X}\left(\mathbb{F}_{q^{m-u}}\right)$, we have $a_{i j} \in \mathbb{F}_{q^{m-u}}$ for all $i, j$, and then $a_{i j}^{1 / q^{m}}=a_{i j}^{1 / q^{u}}$. Thus, setting $w_{i}=$ $\sum_{j=0}^{n} a_{i j}^{1 / q^{u}} f_{j}$ gives $h_{i}=w_{i}^{q^{u}}$ and $b_{i}=w_{i}^{q^{m}}$, and then $\gamma_{l k}=v_{l k}^{q^{u}}$, where $v_{l k}:=w_{k} w_{l}^{q^{m-u}}-w_{l} w_{k}^{q^{m-u}}$. Clearly $P$ is a zero of $v_{l k}$ with $v_{P}\left(v_{l k}\right) \geq j_{1}$. Therefore, $v_{P}\left(\gamma_{l k}\right) \geq j_{1} q^{u}$ and (4.8) with $(l, k)=(n-1, n)$ gives (4.2).

Finally, one can check that the strict inequality conditions $(i),(i i)$ and (iii) are all given by (4.7).

The proof of the following result is analogous to that of [21, Proposition 2.5] and omited accordingly.

Proposition 4.2. Let $P \in \mathcal{X}\left(\mathbb{F}_{q}\right)$ with $(\mathcal{D}, P)$-orders $j_{0}, \ldots, j_{n}$. If $m_{0}, \ldots, m_{n-2}$ are integers such that $0 \leq m_{0}<m_{1}<\cdots<m_{n-2}$ and

$$
\operatorname{det}\left(\left(\begin{array}{c}
j_{i}-j_{2} \\
m_{r}
\end{array}\right)\right)_{0 \leq r \leq n-2,2 \leq i \leq n} \not \equiv 0 \bmod p
$$

then $\kappa_{i} \leq m_{i}$ for all $i$.

Corollary 4.3. Let $P \in \mathcal{X}\left(\mathbb{F}_{q}\right)$. Then $\kappa_{i} \leq j_{i+2}-j_{2}$ for all $i \in\{0,1, \ldots, n-2\}$. Furthermore, $v_{P}\left(T_{u, m}\right) \geq q^{u} j_{1}+j_{2}(n-1)$.

Proof. The first assertion follows from Porposition 4.2 for $m_{i}=j_{i+2}-j_{2}$. The second follows from the first and (4.1).

Theorem 4.4. Let $\mathcal{X}$ be a curve of genus $g$ defined over $\mathbb{F}_{q}$. If $\phi: \mathcal{X} \longrightarrow \mathbb{P}^{n}\left(\overline{\mathbb{F}}_{q}\right)$ is a morphism defined over $\mathbb{F}_{q}$, with $\left(q^{u}, q^{m}\right)$-Frobenius order-sequence $\left(\kappa_{0}, \kappa_{1}, \ldots, \kappa_{n-2}\right)$, then

$$
c_{1} N_{1}+c_{u}\left(N_{u}-N_{1}\right)+c_{m}\left(N_{m}-N_{1}\right)+c_{m-u}\left(N_{m-u}-N_{1}\right) \leq(2 g-2) \cdot \sum_{i=0}^{n-2} \kappa_{i}+\left(q^{m}+q^{u}+n-1\right) d,
$$

where $d$ is the degree of the linear series $\mathcal{D}$ associated to $\phi$ and $c_{r}=\min \left\{v_{P}\left(T_{u, m}\right) \mid P \in \mathcal{X}\left(\mathbb{F}_{q^{r}}\right)\right\}$ for $r \in\{1, u, m, m-u\}$. In addition, $c_{m-u} \geq q^{u}$ and $c_{1} \geq q^{u}+\epsilon_{2}(n-1)$.

Proof. Since $\mathcal{X}\left(\mathbb{F}_{q}\right) \subseteq \mathcal{X}\left(\mathbb{F}_{q^{r}}\right)$ for $r \in\{u, m, m-u\}$, then $c_{1} \geq c_{r}$ and $N_{r} \geq N_{1}$ for each $r$. Moreover, since $\operatorname{gcd}(u, m)=1$, we have $\mathcal{X}\left(\mathbb{F}_{q^{r}}\right) \bigcap_{r \neq s} \mathcal{X}\left(\mathbb{F}_{q^{s}}\right)=\mathcal{X}\left(\mathbb{F}_{q}\right)$, for $r, s \in\{1, u, m, m-u\}$. Thus (4.9) follows from (3.4). Corollary 4.3 and (4.2) imply the last statement.

Remark 4.5. One can subtract $B(P):=v_{P}\left(T_{u, m}\right)-c_{r}$ (resp. $v_{P}\left(T_{u, m}\right)$ ) on the right side of (4.9) for each $P \in \mathcal{X}\left(\mathbb{F}_{q^{r}}\right)$ (resp. for each $\left.P \in \mathcal{X}\right)$, with the values of $v_{P}\left(T_{u, m}\right)$ estimated via Proposition (4.1). 
Corollary 4.6. Assume that $\mathcal{X}$ is $\left(q^{u}, q^{m}\right)$-Frobenius classical with respect to $\phi$. Then

$$
(n-1) N_{u}+(n-1) N_{m}+q^{u} N_{m-u} \leq(n-1)(n-2)(g-1)+d\left(q^{m}+q^{u}+n-1\right)-\sum_{P \in \mathcal{X}\left(\mathbb{F}_{q}\right)} B(P)
$$

where $d$ is the degree of the linear series associated to $\phi$ and $B(P) \geq q^{u}\left(j_{1}-1\right)+\sum_{i=2}^{n}\left(j_{i}-i\right)$.

Note that for $n=m=2$, Proposition 3.4 implies $\kappa_{0}=0$, i.e., the curve is $\left(q, q^{2}\right)$-Frobenius classical. Thus Corollary 4.6 further gives

Corollary 4.7. Let $\mathcal{C}$ be an irreducible plane curve of degree $d>1$ defined over $\mathbb{F}_{q}$. If $N_{i}=\mathcal{C}\left(\mathbb{F}_{q^{i}}\right)$, $i=1,2$, then

$$
(q+1) N_{1}+N_{2} \leq d\left(q^{2}+q+1\right)-\sum_{P \in \mathcal{X}\left(\mathbb{F}_{q}\right)}\left(q^{u}\left(j_{1}-1\right)+\left(j_{2}-2\right)\right) .
$$

Under suitable conditions, one can improve the estimate of Proposition 4.1

Proposition 4.8. Let $\left(\nu_{i}\right)$ and $\left(\mu_{i}\right)$ be the respective $\mathbb{F}_{q^{u}}$-and $\mathbb{F}_{q^{m}}$-Frobenius order-sequences with $\mu_{i}=\epsilon_{i}$, $i=0, \ldots, n-1$ and $\left\{\nu_{0}, \nu_{1}, \ldots, \nu_{n-1}\right\}=\left\{\epsilon_{0}, \epsilon_{1}, \ldots, \epsilon_{n}\right\} \backslash\left\{\epsilon_{k}\right\}$ for some $k \in\{1, \ldots, n-1\}$. If $P \in \mathcal{X}\left(\mathbb{F}_{q^{m}}\right)$ has $(\mathcal{D}, P)$-orders $j_{0}, j_{1}, \ldots, j_{n}$ and $p \nmid \operatorname{det}\left(\left(\begin{array}{c}j_{i} \\ \epsilon_{r}\end{array}\right)\right)_{0 \leq i, r \leq n-1}$, then

$$
v_{P}\left(T_{u, m}\right) \geq j_{n}+\sum_{\substack{i=1 \\ i \neq k}}^{n-1}\left(j_{i}-\epsilon_{i}\right)
$$

Proof. For the sake of notation simplicity, the proof will be limited to the case $k=n-1$. The general case is analogous. Let $t \in \mathbb{F}_{q}(\mathcal{X})$ be local paramter at $P$. Thus we assume that the coordinate functions $f_{i} \in \mathbb{F}_{q}(\mathcal{X})$ are regular at $P \in \mathcal{X}\left(\mathbb{F}_{q^{m}}\right)$ and that $f_{0}=1$. Since $\nu_{n-1}=\epsilon_{n}$, we have

$$
\left|\begin{array}{cccc}
1 & f_{1}^{q^{u}} & \cdots & f_{n}^{q^{u}} \\
1 & f_{1} & \cdots & f_{n} \\
0 & D_{t}^{\left(\epsilon_{1}\right)} f_{1} & \cdots & D_{t}^{\left(\epsilon_{1}\right)} f_{n} \\
\vdots & \vdots & \ddots & \vdots \\
0 & D_{t}^{\left(\epsilon_{n-1}\right)} f_{1} & \cdots & D_{t}^{\left(\epsilon_{n-1}\right)} f_{n}
\end{array}\right|=0
$$

and then

$$
\left(1, f_{1}^{q^{u}}, \cdots, f_{n}^{q^{u}}\right)=\sum_{i=0}^{n-1} \delta_{i} \cdot\left(D_{t}^{\left(\epsilon_{i}\right)}(1), D_{t}^{\left(\epsilon_{i}\right)}\left(f_{1}\right), \cdots, D_{t}^{\left(\epsilon_{i}\right)}\left(f_{n}\right)\right)
$$

for some $\delta_{0}, \delta_{1}, \ldots, \delta_{n-1} \in \mathbb{F}_{q}(\mathcal{X})$. From Proposition 3.1, we have $\left\{\kappa_{0}, \kappa_{1}, \ldots, \kappa_{n-2}\right\}=\left\{\epsilon_{0}, \epsilon_{1}, \ldots, \epsilon_{n-2}\right\}$, 
and from

$$
0 \neq\left|\begin{array}{cccc}
1 & f_{1}^{q^{m}} & \cdots & f_{n}^{q^{m}} \\
1 & f_{1}^{q^{u}} & \cdots & f_{n}^{q^{u}} \\
1 & f_{1} & \cdots & f_{n} \\
0 & D_{t}^{\left(\epsilon_{1}\right)} f_{1} & \cdots & D_{t}^{\left(\epsilon_{1}\right)} f_{n} \\
\vdots & \vdots & \ddots & \vdots \\
0 & D_{t}^{\left(\epsilon_{n-2}\right)} f_{1} & \cdots & D_{t}^{\left(\epsilon_{n-2}\right)} f_{n}
\end{array}\right|= \pm \delta_{n-1} \cdot\left|\begin{array}{cccc}
1 & f_{1}^{q^{m}} & \cdots & f_{n}^{q^{m}} \\
1 & f_{1} & \cdots & f_{n} \\
0 & D_{t}^{\left(\epsilon_{1}\right)} f_{1} & \cdots & D_{t}^{\left(\epsilon_{1}\right)} f_{n} \\
\vdots & \vdots & \ddots & \vdots \\
0 & D_{t}^{\left(\epsilon_{n-1}\right)} f_{1} & \cdots & D_{t}^{\left(\epsilon_{n-1}\right)} f_{n}
\end{array}\right|
$$

we have $v_{P}\left(T_{u, m}\right)=v_{P}\left(\delta_{n-1}\right)+v_{P}(S)$, where $S$ is the Stöhr-Voloch divisor with respect to $\mathbb{F}_{q^{m}}$. Hence [21, Proposition 2.4 (a)] gives

$$
v_{P}\left(T_{u, m}\right) \geq v_{P}\left(\delta_{n-1}\right)+\sum_{i=1}^{n}\left(j_{i}-\epsilon_{i-1}\right) .
$$

Let us proceed to evaluate $v_{P}\left(\delta_{n-1}\right)$. Note that if $\sum_{i=0}^{n} a_{i} X_{i}=0$ is the osculating hyperplane at our point $P \in \mathcal{X}\left(\mathbb{F}_{q^{m}}\right)$, then $v_{P}\left(\sum_{i=0}^{n} a_{i} f_{i}\right)=j_{n}$ gives $a_{i} \neq 0$ for some $i \geq 1$. Without loss of generality, we assume $a_{n} \neq 0$. Thus the uniqueness of the osculating hyperplane implies that $j_{0}, \ldots, j_{n-1}$ are also the $\left(\mathcal{D}^{\prime}, P\right)$ orders with respect to $\mathcal{X} \stackrel{\left(1: f_{1}: \ldots: f_{n-1}\right)}{\longrightarrow} \mathbb{P}^{n-1}$. Removing the last coordinate on the vectors of (4.14), we write

$$
\left[\begin{array}{cccc}
1 & 0 & \cdots & 0 \\
f_{1} & D_{t}^{\left(\epsilon_{1}\right)} f_{1} & \cdots & D_{t}^{\left(\epsilon_{n-1}\right)} f_{1} \\
\vdots & \vdots & \ddots & \vdots \\
f_{n-1} & D_{t}^{\left(\epsilon_{1}\right)} f_{n-1} & \cdots & D_{t}^{\left(\epsilon_{n-1}\right)} f_{n-1}
\end{array}\right]\left[\begin{array}{c}
\delta_{0} \\
\delta_{1} \\
\vdots \\
\delta_{n-1}
\end{array}\right]=\left[\begin{array}{c}
1 \\
f_{1}^{q^{u}} \\
\vdots \\
f_{n-1}^{q^{u}}
\end{array}\right]
$$

The function $\delta_{n-1}$ will be obtained via Cramer's rule in (4.17). To this end, we consider the following two determinants associated to $\mathcal{X} \stackrel{\left(1: f_{1}: \ldots: f_{n-1}\right)}{\longrightarrow} \mathbb{P}^{n-1}$ :

$$
A=\left|\begin{array}{cccc}
1 & f_{1} & \cdots & f_{n-1} \\
0 & D_{t}^{\left(\epsilon_{1}\right)} f_{1} & \cdots & D_{t}^{\left(\epsilon_{1}\right)} f_{n-1} \\
0 & D_{t}^{\left(\epsilon_{2}\right)} f_{1} & \cdots & D_{t}^{\left(\epsilon_{2}\right)} f_{n-1} \\
\vdots & \vdots & \ddots & \vdots \\
0 & D_{t}^{\left(\epsilon_{n-1}\right)} f_{1} & \cdots & D_{t}^{\left(\epsilon_{n-1}\right)} f_{n-1}
\end{array}\right| \text { and } B=\left|\begin{array}{cccc}
1 & f_{1}^{q^{u}} & \cdots & f_{n-1}^{q^{u}} \\
1 & f_{1} & \cdots & f_{n-1} \\
0 & D_{t}^{\left(\epsilon_{1}\right)} f_{1} & \cdots & D_{t}^{\left(\epsilon_{1}\right)} f_{n-1} \\
\vdots & \vdots & \ddots & \vdots \\
0 & D_{t}^{\left(\epsilon_{n-2}\right)} f_{1} & \cdots & D_{t}^{\left(\epsilon_{n-2}\right)} f_{n-1}
\end{array}\right| \text {. }
$$

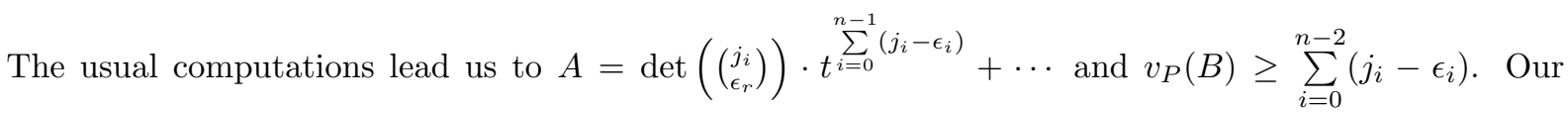


hypothesis $\operatorname{det}\left(\left(\begin{array}{c}j_{i} \\ \epsilon_{r}\end{array}\right)\right) \neq 0$ further gives $v_{P}(A)=\sum_{i=0}^{n-1}\left(j_{i}-\epsilon_{i}\right)$. Hence Cramer's rule yields

$$
v_{P}\left(\delta_{n-1}\right)=v_{P}(B / A) \geq \sum_{i=0}^{n-2}\left(j_{i}-\epsilon_{i}\right)-\sum_{i=0}^{n-1}\left(j_{i}-\epsilon_{i}\right)=-\left(j_{n-1}-\epsilon_{n-1}\right)
$$

which, together with (4.16), gives the result.

Remark 4.9. Note that under the conditions of Proposition 4.8, we have that $\epsilon_{k+1}=p^{r}$ for some $r>0$.

Proposition 4.10. Consider $\mathcal{X}$ with the hypotheses of Proposition 4.8 such that $p \nmid \operatorname{det}\left(\left(\begin{array}{c}j_{i}(P) \\ \epsilon_{r}\end{array}\right)\right)_{0 \leq i, r \leq n-1}$ for all $P \in \mathcal{X}\left(\mathbb{F}_{q^{m}}\right) \backslash \mathcal{X}\left(\mathbb{F}_{q}\right)$. Then

$$
\epsilon_{k} N_{u}+\epsilon_{n} N_{m}+q^{u} N_{m-u} \leq(2 g-2) \cdot \sum_{\substack{i=1 \\ i \neq k}}^{n-1} \epsilon_{i}+d\left(q^{m}+q^{u}+n-1\right)
$$

where $d=\operatorname{deg}(\mathcal{D})$.

Proof. The hypotheses of Proposition 4.8 provide $\left\{\kappa_{0}, \ldots, \kappa_{n-2}\right\}=\left\{\epsilon_{0}, \ldots, \epsilon_{n-1}\right\} \backslash\left\{\epsilon_{k}\right\}$ and $\left\{\nu_{0}, \ldots, \nu_{n-1}\right\}=$ $\left\{\kappa_{0}, \ldots, \kappa_{n-2}\right\} \cup\left\{\epsilon_{n}\right\}$. In particular, $\epsilon_{i} \geq \kappa_{i-1}$ for all $i \in\{1, \ldots, n-1\}$ and $\epsilon_{n}=\nu_{n-1}$. Let $P \in \mathcal{X}\left(\mathbb{F}_{q^{u}}\right)$. Then Proposition 4.1 implies that

$$
v_{P}\left(T_{n, m}\right) \geq \sum_{i=1}^{n-1}\left(j_{i}(P)-\kappa_{i-1}\right) \geq \epsilon_{k}
$$

Moreover, by [21, Corollary 2.6], we have $j_{n}(P) \geq \epsilon_{n}+j_{1}(P)$, and then

$$
v_{P}\left(T_{n, m}\right) \geq q^{u} j_{1}(P)+\sum_{i=0}^{n-2}\left(j_{i+2}(P)-\kappa_{i}\right) \geq q^{u} j_{1}(P)+\epsilon_{n}+j_{1}(P)+\sum_{i=2}^{n-1}\left(j_{i}(P)-\kappa_{i-1}\right)
$$

for all $P \in \mathcal{X}\left(\mathbb{F}_{q}\right)$. If $P \in \mathcal{X}\left(\mathbb{F}_{q^{m-u}}\right)$, Proposition 4.1 gives $v_{P}\left(T_{u, m}\right) \geq q^{u} j_{1}(P)$, and if $P \in \mathcal{X}\left(\mathbb{F}_{q^{m}}\right)$, then $v_{P}\left(T_{u, m}\right) \geq j_{n}(P)$ by Proposition 4.8, Thus (4.19) follows from Theorem 4.4.

Corollary 4.11. Let $\mathcal{F}$ be an irreducible, $\mathbb{F}_{q^{u}}$-Frobenius nonclassical plane curve of degree d. If $j_{1}(P) \not \equiv 0$ $\bmod p$ for all $P \in \mathcal{X}\left(\mathbb{F}_{q^{m}}\right) \backslash \mathcal{X}\left(\mathbb{F}_{q}\right)$, then

$$
\epsilon_{2} N_{m}+q^{u} N_{m-u} \leq\left(q^{m}+d-1\right) d
$$

Proof. Since $\mathcal{F}$ is $\mathbb{F}_{q^{u}}$-Frobenius nonclassical, it follows from [4, Corollary 1.4] that $N_{u} \geq d\left(q^{u}-d+2\right)$. Hence we obtain (4.20) directly from Proposition 4.10.

Remark 4.12. As in Theorem 4.4, bound (4.19) can be improved according to the values $B(P), P \in \mathcal{X}$. Assuming $m=2$ and taking into account the values $B(P)$ for $P \in \mathcal{X}\left(\mathbb{F}_{q}\right)$, we obtain the following. 
Proposition 4.13. Consider $\mathcal{X}$ with the hypotheses of Proposition 4.8 with $m=2$. Assume that that

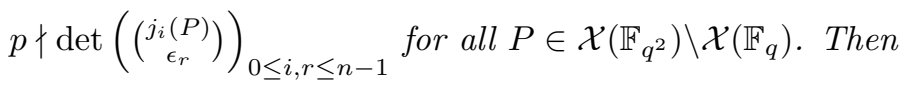

$$
\left(q+\epsilon_{k}\right) N_{1}+\epsilon_{n} N_{2} \leq(2 g-2) \cdot \sum_{\substack{i=1 \\ i \neq k}}^{n-1} \epsilon_{i}+d\left(q^{2}+q+n-1\right)-\sum_{P \in \mathcal{X}\left(\mathbb{F}_{q}\right)} B(P)
$$

where $d=\operatorname{deg}(\mathcal{D})$ and $B(P) \geq q\left(j_{1}(P)-1\right)-j_{1}(P)+\sum_{i=1}^{n}\left(j_{i}(P)-\epsilon_{i}\right)$. In particular, if $n=2$,

$$
(q+1) N_{1}+\epsilon_{2} N_{2} \leq d\left(q^{2}+q+1\right)-\sum_{P \in \mathcal{X}\left(\mathbb{F}_{q}\right)}\left(q\left(j_{1}(P)-1\right)+j_{2}(P)-\epsilon_{2}-j_{1}(P)\right) .
$$

\section{Examples}

The effectiveness of the bounds presented in the previous sections can be verified in many cases. This section illustrates cases found in the literature.

Example 5.1. For irreducible plane curves of degree $d \geq 2$, defined over $\mathbb{F}_{q}$, Corollary 4.7 gives

$$
(q+1) N_{1}+N_{2} \leq\left(q^{2}+q+1\right) d-\sum_{P \in \mathcal{X}\left(\mathbb{F}_{q}\right)}\left(j_{2}(P)-2\right) .
$$

This bound is clearly sharp for conics, but one can certainly find less obvious cases of sharpness. For instance, for $q \equiv 0 \bmod 3$, the Fermat curve over $\mathbb{F}_{q}$

$$
\mathcal{X}: x^{q-1}+y^{q-1}+z^{q-1}=0
$$

has no $\mathbb{F}_{q}$-inflection point; $N_{1}=(q-1)^{2}$ and $N_{2}=3(q-1)+(q-1)^{2}$ (see ([18, Theorem 1]).

Example 5.2. Bound (5.1) may be of interest, even if we focus on $N_{1}$. In fact, since $N_{1} \leq N_{2}$, we have

$$
N_{1} \leq\left(q-1+\frac{3}{q+2}\right) d
$$

Curiously, a bound similar to (5.2) was proved by Homma and Kim in [14]1 However, in their context, $N_{1}$ is the number of points $P \in \mathbb{P}^{2}\left(\mathbb{F}_{q}\right)$ on a plane curve defined over $\mathbb{F}_{q}$, without $\mathbb{F}_{q}$-linear components. Therefore, in the singular setting, the two bounds have different meanings. Examples of curves attaing (5.2) can be easily found in the literature. For instance, the smooth curves over $\mathbb{F}_{q}$

$$
y\left(y^{q} z-y z^{q}\right)+z\left(z^{q} x-z x^{q}\right)+(a x+b y+c z)\left(x^{q} y-x y^{q}\right)=0,
$$

\footnotetext{
${ }^{1}$ Let $f(x, y, z) \in \mathbb{F}_{q}[x, y, z]$ be a homogeneous polynomial of degree $d$ without $\mathbb{F}_{q}$-linear factor. In [22], Tallini proved that if $R_{q} \geq q^{2}+q+1$, then $d \geq q+2$, where $R_{q}$ denotes the number of solutions of $f(x, y, z)=0$ in $\mathbb{P}^{2}\left(\mathbb{F}_{q}\right)$. It follows from bound (5.2) that the same result holds when we replace $R_{q}$ by $N_{1}$.
} 
where $a, b, c \in \mathbb{F}_{q}$ are such that $t^{3}-\left(c t^{2}+b t+a\right) \in \mathbb{F}_{q}[t]$ is irreducible (see [15]).

Bound (5.2) can be slightly improved if rational inflection points occur. For instance, suppose $\mathcal{X}$ is a smooth plane curve of degree $d$ and $P \in \mathcal{X}\left(\mathbb{F}_{q}\right)$ is a total inflextion point, that is, $j_{2}(P)=d$. Thus (5.1) reads $(q+1) N_{1}+N_{2} \leq\left(q^{2}+q+1\right) d-(d-2)$, and then $N_{1} \leq N_{2}$ gives

$$
N_{1} \leq \frac{\left(q^{2}+q\right) d+2}{q+2}
$$

The smooth plane curve $x^{q+1}-x^{2} z^{q-1}+x^{q} z-y z^{q}=0$ over $\mathbb{F}_{q}$ has a total inflection point, namely $P=(0: 1: 0)$, and attains bound (5.3), as $N_{1}=q^{2}+1$ (see [14]).

In the latter examples, tantamount to attaining the upper bound for $N_{1}$ is concluding via (5.1) that $N_{2}=N_{1}$. These small values for $N_{2}$ could not be predicted by classical bounds such as Weil's, StöhrVoloch's, or Ihara's bound in [16].

Example 5.3. One can also take advantage of the bounds if there is prior knowledge of, say, $N_{1}$. Some Frobenius nonclassical curves will be used to illustrate this. If $\mathcal{C}$ is a plane $\mathbb{F}_{q}$-Frobenius nonclassical curve then, from Theorem 2.2, $\mathcal{C}$ is $\mathbb{F}_{q^{2}}$-Frobenius classical. Thus if $p \nmid j_{1}(P)$ for all $P \in \mathcal{C}\left(\mathbb{F}_{q^{2}}\right) \backslash \mathcal{C}\left(\mathbb{F}_{q}\right)$, then Proposition 4.13 gives

$$
(q+1) N_{1}+\epsilon_{2} N_{2} \leq d\left(q^{2}+q+1\right)-\sum_{P \in \mathcal{X}\left(\mathbb{F}_{q}\right)}\left(q\left(j_{1}(P)-1\right)+j_{2}(P)-\epsilon_{2}-j_{1}(P)\right) .
$$

For the $\mathbb{F}_{q^{3}}$-Frobenius nonclassical curve (see [1, [8, [10])

$$
\mathcal{Y}_{q, 3}: x^{q^{2}+q+1}+y^{q^{2}+q+1}=1
$$

it is known that $N_{1}:=\# \mathcal{Y}_{q, 3}\left(\mathbb{F}_{q^{3}}\right)=q^{5}-q^{3}-q^{2}+1$ and $\epsilon_{2}=q$. The $3\left(q^{2}+q+1\right)$ inflection points $P_{i} \in \mathcal{F}\left(\mathbb{F}_{q^{3}}\right)$ have order-sequence $\left(j_{0}, j_{1}, j_{2}\right)=\left(0,1, q^{2}+q+1\right)$, and then $B\left(P_{i}\right) \geq q^{2}$. Using these data, bound (5.4) gives $N_{2} \leq q^{6}+2 q^{5}+q^{4}-2 q^{3}-2 q^{2}-q+1$. Similarly, one can bound $N_{2}:=\# \mathcal{F}\left(\mathbb{F}_{q^{6}}\right)$ for the Norm-Trace curve

$$
\mathcal{X}_{q, 3}: x^{q^{2}+q+1}=y^{q^{2}}+y^{q}+y
$$

and arrive at $N_{2} \leq q^{6}+q^{5}-q^{3}+1$. It can be checked that these bounds are better than Weil's, StöhrVoloch's and Ihara's bounds. In fact, it is not difficult to prove that these are the actual values for $N_{2}$.

Example 5.4. For an example where $\mathcal{X} \hookrightarrow \mathbb{P}^{n}$ and $n>2$, consider the Fermat curve

$$
\mathcal{F}: x^{\frac{q+1}{2}}+y^{\frac{q+1}{2}}=1
$$

over $\mathbb{F}_{q^{2}}$ with $q \not \equiv 0$ mod 5. This curve is a well-known $\mathbb{F}_{q^{2}}$-maximal curve of genus $g=\frac{(q-1)(q-3)}{8}$. In particular, $N_{1}=1+q^{2}+2 g q$ and $N_{2}=1+q^{4}-2 g q^{2}$. In addition, $\mathcal{F}$ is $\mathbb{F}_{q^{2}}$-Frobenius nonclassical 
for the morphism of conics $\mathcal{F} \hookrightarrow \mathbb{P}^{5}$ (see [8]). Simple manipulation of equation (5.5) will give us that $\mathcal{C}_{P}: g_{P}(x, y)=0$, where

$$
g_{P}(x, y)=\left(a^{q} \cdot x+b^{q} \cdot y-1\right)^{2}-4(a b)^{q} x y
$$

is the osculating conic at a general point $P=(a, b) \in \mathcal{F}$. Since $\mathcal{F}$ is classical for the morphism of lines, it follows that $\epsilon_{i}=i$ for $i \leq 4$ and $\epsilon_{5}=q$. While the $\mathbb{F}_{q^{2}}$-Frobenius map clearly takes $P$ to $\mathcal{C}_{P}$, i.e., $g_{P}\left(a^{q^{2}}, b^{q^{2}}\right)=0$, this is not the case for the $\mathbb{F}_{q^{4}}$-Frobenius map, that is, the function

$$
\left(x^{q} \cdot x^{q^{4}}+y^{q} \cdot y^{q^{4}}-1\right)^{2}-4(x y)^{q}(x y)^{q^{4}}
$$

does not vanish. Thus $\mathcal{F}$ is $\mathbb{F}_{q^{4}}$-Frobenius classical. In additon, since all inflection points lie in $\mathcal{F}\left(\mathbb{F}_{q^{2}}\right)$, it follows that $\operatorname{det}\left(\left(\begin{array}{c}j_{i}(P) \\ \epsilon_{r}\end{array}\right)\right)_{0 \leq i, r \leq 4}=1$ for all $P \in \mathcal{F}\left(\mathbb{F}_{q^{4}}\right) \backslash \mathcal{F}\left(\mathbb{F}_{q^{2}}\right)$. Therefore, Proposition 4.13 gives

$$
\left(q^{2}+\epsilon_{4}\right) N_{1}+\epsilon_{5} N_{2} \leq(2 g-2) \cdot \sum_{i=1}^{3} \epsilon_{i}+d\left(q^{4}+q^{2}+4\right)-\sum_{P \in \mathcal{X}\left(\mathbb{F}_{q^{2}}\right)} B(P)
$$

Note that each of the $3(q+1) / 2$ inflection points $P_{i}$ have order-sequence $(0,1,2,(q+1) / 2,(q+3) / 2, q+1)$, and then $B\left(P_{i}\right) \geq q-5$. Plugging the corresponding values in (5.6), we see that it becames an equality.

Example 5.5. To conclude examples, consider the Hermitian curve

$$
\mathcal{H}: x^{q+1}+y^{q+1}=1
$$

over $\mathbb{F}_{q^{2}}$. The curve $\mathcal{H}$ is $\mathbb{F}_{q^{2}}$-Frobenius nonclassical with respect to the morphism of lines, and $\epsilon_{2}=q$. Since $\mathcal{H}$ is $\mathbb{F}_{q^{2}}$-maximal of genus $g=q(q-1) / 2$, we have $N_{m}=q^{2 m}+1+(-1)^{m-1} q^{m+1}(q-1)$ (see e.g. [13, p.336]). Thus

$$
q N_{m}+q^{2} N_{m-1}=d\left(q^{2 m}+d-1\right),
$$

where $d=q+1$ is the degree of $\mathcal{H}$. That is, $\mathcal{H}$ meets the bound in Corollary (4.11).

\section{Acknowledgments}

The first author was partially supported by FAPESP-Brazil, grant 2013/00564-1.

\section{References}

[1] H. Borges, Frobenius nonclassical components of curves with separated variables, Journal of Number Theory, v. 159 (2016), 402-426.

[2] H. Borges, On complete $(N, d)$-arcs derived from plane curves, Finite Fields Appl., 15(2009) 82-96. 
[3] H. Borges, On multi-Frobenius non-classical plane curves, Arch. Math. (Basel) 93(2009), no. 6, 541553.

[4] H. Borges, M. Homma, Points on singular Frobenius nonclassical curves, http://arxiv.org/abs/1511.00339 (2015).

[5] M.L. Carlin, J.F. Voloch, Plane curves with many points over finite fields, Rocky Montain J. Math., $34(2004)$ 1255-1259.

[6] A. Garcia, The curves $y^{n}=f(x)$ over finite fields, Arch. Math. 54(1990) 36-44.

[7] A. Garcia, J.F. Voloch, Wronskians and linear independence in fields of prime characteristic, Manuscripta Math. 59(1987) 457-469.

[8] A. Garcia, J.F. Voloch, Fermat Curves over finite fields, J. Number Theory 30(1988) 345-356.

[9] V.D. Goppa, Codes on algebraic curves, Soviet Math. Dokl. 24 No.1(1980) 170-172.

[10] A. Hefez, J.F. Voloch, Frobenius non-classical curves, Arch. Math. 54 (1990) 263-273.

[11] J.W.P. Hirschfeld, Projective geometries over finite fields, 2nd edn, Oxford University Press, 1998.

[12] J.W.P. Hirschfeld, G. Korchmáros, On the number of rational points on an algebraic curve over a finite field, Bull. Belg. Math. Soc. 5(1998) 313-340.

[13] J.W.P. Hirschfeld, G. Korchmáros, F.Torres, Algebraic curves over a finite field, Princeton Series in Applied Mathematics, 2008.

[14] M. Homma and S. J. Kim, Around Sziklai's conjecture on the number of points of a plane curve over a finite field, Finite Fields Appl. 15 (2009) 468-?474.

[15] M. Homma and S. J. Kim, Nonsingular plane filling curves of minimum degree over a finite field and their automorphism groups: Supplements to a work of Tallini .

[16] Y. Ihara, Some Remarks on the number of rational points of algebraic curves over finite fields, J. Fac. Sci. Univ. Tokyo, Sect. IA Math. 28(1981) 721-724.

[17] R. Lidl, H. Niederreiter, Finite fields, Cambridge University Press, 1988.

[18] M. Moisio, On the number of rational points on some families of Fermat curves over finite fields, Finite Fields Appl. 13 (2007) 546-562.

[19] J-P. Serre, Sur le noumbre des points rationnels d'une courbe algébrique sur un corps fini, C.R. Acad. Sci. Paris Sér I Math., 296(1983) 397-402.

[20] H. Stichtenoth, Algebraic function fields and codes, Springer, Berlin, 1993. 
[21] K.O. Stöhr, J.F. Voloch, Weierstrass points and curves over finite fields, Proc. London Math. Soc. 52(1986) 1-19.

[22] G. Tallini, Sulle ipersuperficie irriducibili d'ordine minimo che contengono tutti i punti di uno spazio di Galois $S_{r, q}$, Rend. Mat. Roma 20(1961) 431-479.

[23] F. Rodriguez Villegas, J.F. Voloch, D. Zagier, Constructions of plane curves with many points, Acta Arith. 99(2001) 85-96.

[24] A. Weil, Courbes algébriques et variétés abéliennes (Hermann, Paris, 1971). 